\title{
Correction of parameters of fiber-optical systems on the basis of the magneto tunable gradient elements
}

\author{
Leonovich G.I., \\ Samara State Aerospace University \\ Karpeev S.V., \\ Samara State Aerospace University \\ Institute of Image Processing Systems, Russian Academy of Sciences \\ Paranin V.D. \\ Samara State Aerospace University
}

\begin{abstract}
A method of controlling optical parameters of elements and structures formed inside step-index and gradient optic fibers on the basis of external magnetostrictive impact on physical and geometric parameters of light guides is proposed in the paper. The control is accomplished by the impact of a 3D-magnetic actuator on the magnetosensitive thin-film coating of optic fiber.
\end{abstract}

Keywords: gradient optic fibers, 3D-magnetic actuator, thin-film coating of optic fiber.

Citation: Leonovich G.I., Karpeev S.V., Paranin V.D. Correction of parameters of fiber-optical systems on the basis of the magneto tunable gradient elements. Proceedings of Information Technology and Nanotechnology (ITNT-2015), CEUR Workshop Proceedings, 2015; 1490: 133-137. DOI: 10.18287/16130073-2015-1490-133-137

\section{Introduction}

The latest achievements in the area of fiber-optic technologies are widely used today to form high-speed stable data links [1] and intelligent sensor-based systems [2].

When high-speed fiber-optic data links are formed the impact of temperature and mechanical strain $[3,4]$ on the refracting index, ellipticity and length of optic fiber (OF) in the process of cabling should be taken into account and controlled. To avoid mechanical tension and strain of $\mathrm{OF}$ special cable structures are used for instance, wavy profile structures or those with modular stranding. Nevertheless, thermal effect still leads to local deformation of $\mathrm{OF}$ in all structures. As a result, the speed of information transfer may decrease by a factor of two or more. Additional noise, attenuation, modal dispersion $[1,5]$ and crosstalk appear in the cable, which also has a negative impact on the range and quality of communication, including that in the case 
of information transfer from fiber-optic sensors [2]. Methods of controlling these phenomena focused on the use of mode filtration [5-10] are known, but those are rather complex and expensive technologies. The use of optic vortices to transmit information along fiber light guides [11, 12] or dispersion-free beams [13] is another way of correcting distortion, but these methods do not allow transmitting information to considerable distances.

Therefore, the search and application of efficient measures of on-line control of OF incorporated in fiber-optic systems, quick trimming and correction of parameter distortion caused by the impact of operational factors remain a pressing challenge that is not entirely solved today.

\section{Physical foundations of the proposed solution}

The method proposed is based on the combination of the force field forming unit with OF incorporated in its body and an optional sensitive film coating. This device represents a 3D-actuator for the variation of geometrical and related physical and optical OF parameters.

The actuator can be realized in the versions of control by temperature and direct mechanical impact, as well as indirect impact of force magnetic and/or electric field. From the point of view of energy costs, dynamic characteristics, hysteresis phenomena, dependence of the impact on the distance and possibility of constructing systems with fixed parameters control by the external magnetic field is the preferable option.

The main OF optical parameters in the context of the actuator's functional area are the distribution of the refracting index that determines the OF optical properties and the optical path length.

The effects of a control system based on a 3D-magnetic actuator (3DMA) realized within the limits of mechanical strain admissible for a specific type of OF are:

- general and local control of the OF optical path length;

- general and local control of the OF refracting index distribution;

- control of $\mathrm{OF}$ anisotropic properties, including ellipticity and birefringence;

- creation of simple and complex gradient structures with constant and variable refraction parameters;

- control of the spatial position of fiber ends and local areas of OF;

- control of intrafibrous Bragg grating parameters.

\section{Realizable aspects of the proposed technology}

Versions of structural arrangement of the control system for different formats and position of controlled elements are presented in fig. 1 and 2. The gradient magnetic field forming unit is responsible for the formation of a structure with OF local axial and radial non-uniformities (fig. 1). In the general case the unit contains a group of $N$ operating solenoids and $M$ permanent magnets wherein the appropriate $\mathrm{OF}$ areas with a magnetostrictive coating act as armature or magnets interacting with it. It is possible to use materials possessing magnetostrictive properties the use of which in many applications is limited by low sensitivity to the impinging magnetic field. A polymer 
coating is used to produce microdisplacements of areas of optic fiber and permanent magnets are mounted on it.

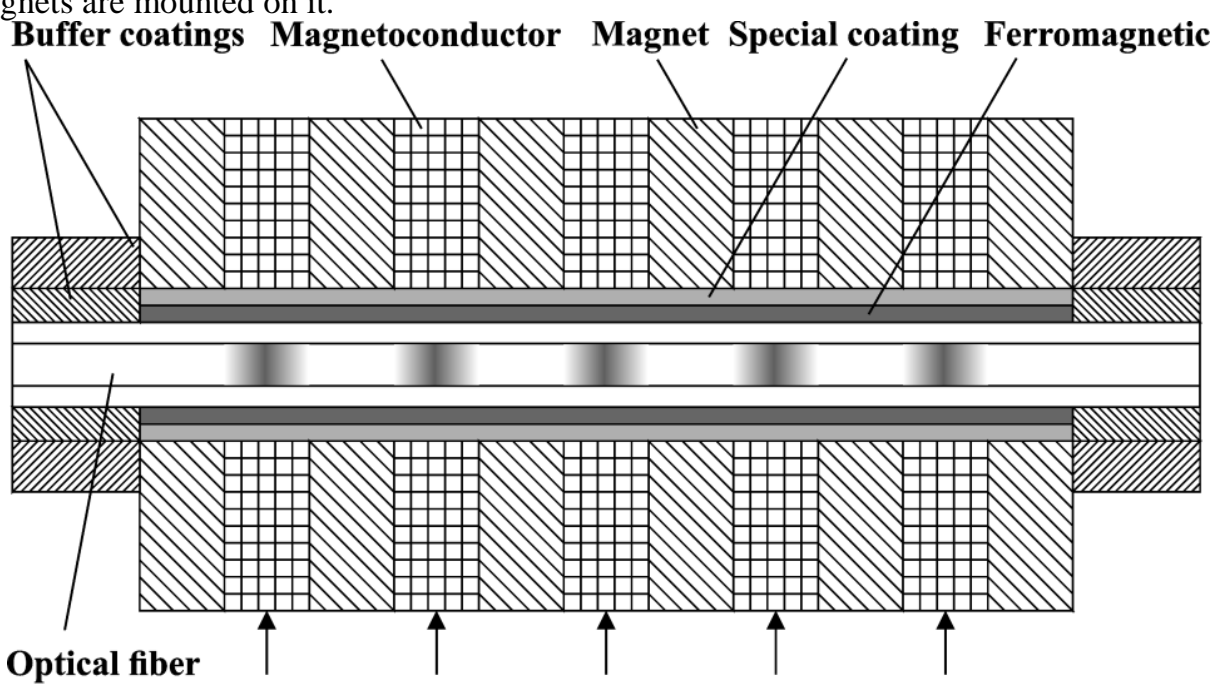

Signals of controlling system

Fig. 1. - Scheme of a multiple module localized system for controlling the properties of an OF area

Fig. 2 presents a structure based on a set of actuators spaced along the OF length on permanent magnets with the function of fixed axial and radial local-integral extension and compression. It is intended to form a gradient structure in an OF specimen.

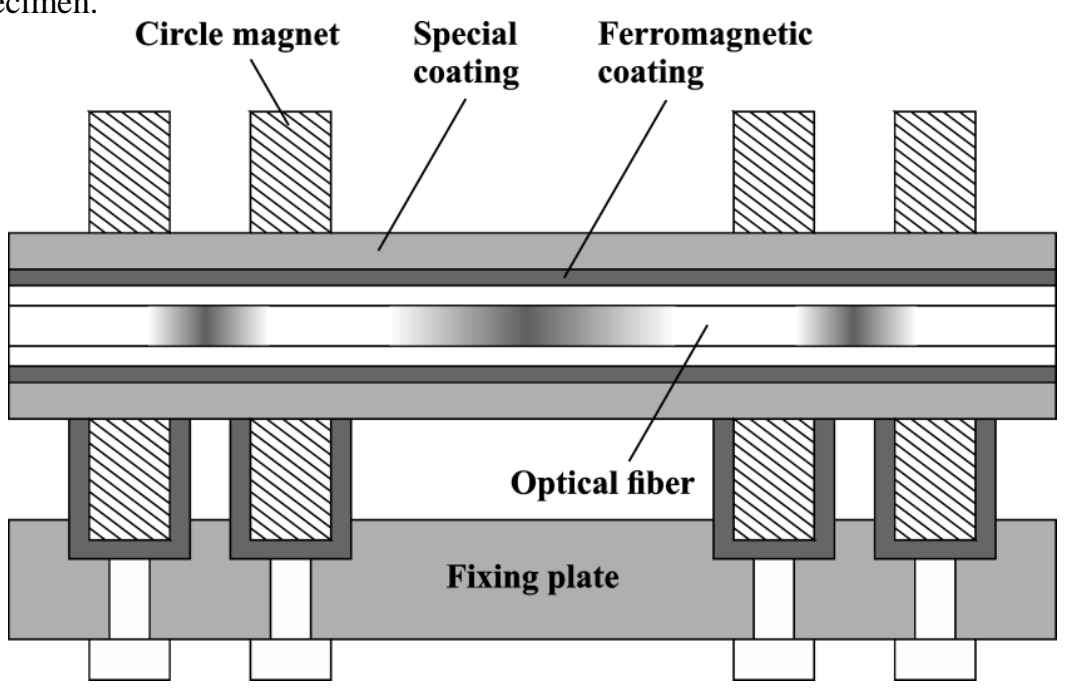

Fig. 2. - Scheme a fixed in-fiber optical structure based on a set of permanent magnet actuators spaced along the OF length 
Local axial compression and extension in a device of this kind takes place due to the interaction of fixed or shifting permanent magnets. Local axial compression takes place in the OF area between permanent annular magnets $2(+)$ and $3(-)$, while local axial extension takes place in the area between the magnets $1(-)$ and $2(-), 3(+)$ and 4 $(+)$. Axial and radial compression and extension is also caused by the interaction of annular and other magnets with a magnetosensitive coating deposited on the OF. The choice of the arrangement, material, selection and arrangement of the magnets are also determined by calculation according to the results of mathematic simulation.

The structures presented as examples can be used both for connected step-index profile OFs and for those with gradient variation of the refracting index.

There are two lines of possible usage for step-index profile OFs: first, to control OF parameters essential for the formation of high-speed data links- the refracting index, anisotropic and other properties mentioned above, and, second, to implement a new principle of creating a gradient structure in a step-index OF. In the case of radial extension or compression realized by the described actuator structure variation of the refracting index of the OF material is approximately proportional to the square of the radius. Consequently, this variation is expected to result in the origination of lenslike gradient medium, that is, in the formation of a new optical element- a gradient lens with a tuning option. A positive lens is formed in the case of radial extension and a negative one in the case of compression.

If $\mathrm{OF}$ with gradient variation of the refracting index is used the control of the external magnetic field takes place in the form of tuning or correction of the parameters of the available lenslike medium.

\section{Conclusion}

The prospects of the implementation of the proposed approach arise from the following factors:

- the possibility of creating a wide range of hybrid-gradient intrafibrous structures that combine the properties of controlled gradient OF medium and the properties of refractive, diffractive and nonlinear-optical elements (gradient lenses, integraloptic, phase and amplitude phase structures);

- the use of the mastered technologies of producing basic components (optical fiber, magnet actuators for microdisplacement of objects);

- the use of simple production equipment and unified techniques to produce elements;

- the simplicity of elements assembly and operation;

- high reliability of the components, resistance to external destabilizing factors.

\section{Acknowlegements}

This work was financially supported by the Russian Ministry of Education and Science. 


\section{References}

1. Kutluyarov RV, Sultanov AH, Bagmanov VH. Reduction of WDM-transmission nonlinear impairments due to polarization mode dispersion. Computer Optics, 2014; 38(4): 737-742.

2. Karpeev SV, Pavelyev VS, Khonina SN, Kazanskiy NL, Gavrilov AV, Eropolov VA. Fiber sensors based on transverse mode selection. Journal of Modern Optics, 2007; 54(6): 833-844.

3. Garitchev VP, Golub MA, Karpeev SV, Krivoshlykov SG, Petrov NI, Sissakian IN, Soifer VA, Haubenreisser W, Jahn JU, Willsch R. Experimental investigation of mode coupling in a multimode graded-index fiber, caused by periodic microbends using computer-generated spatial filters. Optics Communication, 1985; 55(6): 403-405.

4. Karpeev SV, Pavelyev VS, Khonina SN. Investigation of dependence of mode intensity at the exit of the step-index optical fiber from its deformation. Computer Optics, 2003; 25: 95-99. [in Russian]

5. Lyubopytov VS, Tlyavlin AZ, Sultanov AKh, Bagmanov VKh, Khonina SN, Karpeev SV, Kazanskiy NL. Mathematical model of completely optical system for detection of mode propagation parameters in an optical fiber with few-mode operation for adaptive compensation of mode coupling. Computer Optics, 2013; 37(3): 352-359.

6. Golub MA, Kazanskiy NL, Sissakian IN,Soifer VA, Karpeev SV, Mirzov AV,Uvarov GV. Spatial phase filters matched to transver modes. Quantum Electronics, 1988; 18(3): 392-393.

7. Karpeev SV, Pavelyev VS, Duparre M, Luedge B, Rockstuhl C, Schroeter S. DOEaided analysis and generation of transverse coherent light modes in a stepped-index optical fiber. Optical Memory and Neural Networks (Information Optics), 2003; 12(1): 27-34.

8. Golub MA, Karpeev SV, Krivoshlykov SG, Prokhorov AM, Sisakyan IN, Soifer VA. Spatial filter investigation of the distribution of power between transverse modes in a fiber waveguide. Quantum Electronics, 1984; 11(9): 1869-1871.

9. Garitchev VP, Golub MA, Karpeev SV, Krivoshlykov SG, Sissakian IN, Soifer VA, Uvarov GV. Application of the synthesized holograms for selective excitement of gradient optical fiber modes and investigation of their sensitivity to radial shift of an exciting bunch. Computer Optics, 1988; 3: 103-109. [in Russian]

10. Karpeev SV, Pavelyev VS, Duparre M, Ludge B, Schroeter S. Mode excitement of a step-index waveguide by means of binary phase DOE. Computer Optics, 2002; 24: 99-101. [in Russian]

11. Karpeev SV, Khonina SN. Experimental excitation and detection of angular harmonics in a step-index optical fiber. Optical Memory \& Neural Networks (Information Optics), 2007; 16(4): 295-300. [in Russian]

12. Khonina SN, Karpeev SV. Excitation and detecting angular harmonics in fiber waveguide using DOE. Computer Optics, 2004; 26: 16-26. [in Russian]

13. Borodin SA, Volkov AV, Kazanskiy NL, Pavelyev VS, Karpeev SV, Palagushkin AN, Prokopenko SA, Sergeev AP, Arlamenkov AN. Numerical and experimental study of the dispersion-free multimode beams formed by means of DOE. Computer Optics, 2005; 27: 41-44. [in Russian] 